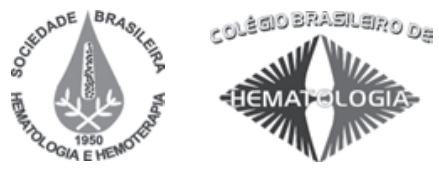

\title{
Púrpura trombocitopênica imune da criança: experiência de 12 anos em uma única instituição brasileira
}

\author{
Childhood immune thrombocytopenic purpura: 12-year experience of a single \\ Brazilian institution
}

Raquel B. Delgado ${ }^{1}$

Marcos B. Viana ${ }^{2}$

Rachel A. F. Fernandes ${ }^{3}$
O objetivo deste trabalho foi determinar a frequência dos quadros clínicos da púrpura trombocitopênica imune e sua associação com contagem de plaquetas, taxa de resposta à esplenectomia e fatores preditivos do desfecho e da evolução para a cronicidade. Realizou-se estudo retrospectivo com 187 crianças diagnosticadas no Hospital das Clínicas da UFMG, entre 04/1988 e 12/2001. Quadros assintomáticos e leves corresponderam a $76 \%$ do total. Hemorragias exclusivamente cutâneas ocorreram em $96 \%$ dos casos sintomáticos. A gravidade dos sintomas associou-se à intensidade da plaquetopenia. Evolução aguda foi apresentada por 123 pacientes (70,7\%) e crônica por 51 (29,3\%). A apresentação insidiosa (26,2\%) associou-se a um maior número de esplenectomias $\left(p=4 \times 10^{-7}\right)$, a uma menor taxa de resposta à corticoterapia $(p=0,003)$ e constituiu-se, juntamente com a ausência de resposta à corticoterapia $\left(p<1 \times 10^{-7}\right)$, em fator preditivo da cronicidade $\left(p=1 \times 10^{-7}\right)$. Taxa de resposta de $74,5 \%$ foi alcançada com a esplenectomia. Remissão foi o desfecho final em $80,2 \%$ dos pacientes. Foram preditivos de remissão final: gênero masculino $(p=0,02)$, número baixo de plaquetas ao diagnóstico $(p=0,004)$, resposta à corticoterapia $(p=0,003)$ e ocorrência de uma primeira remissão $\left(p<1 \times 10^{-7}\right)$. Confirmou-se que a doença na criança é benigna e autolimitada. Houve associação entre gravidade dos sintomas e intensidade da plaquetopenia. Constituíram-se fatores preditivos da cronicidade: apresentação insidiosa e ausência de resposta à corticoterapia. Constituíram-se fatores associados à remissão final: gênero masculino, número mais baixo de plaquetas ao diagnóstico, resposta à corticoterapia inicial e ocorrência de uma primeira remissão. Rev. Bras. Hematol. Hemoter. 2009;31(1):29-36.

Palavras-chave: Púrpura trombocitopênica imune; púrpura trombocitopênica idiopática; plaquetopenia; criança.

\section{Introdução}

A púrpura trombocitopênica imune ou idiopática ou imune primária (PTI) é uma das doenças hemorrágicas adquiridas mais comuns da infância, ocorrendo numa frequência de 4 a 8 casos por 100 mil crianças por ano. Usualmente, é uma doença benigna, autolimitada e ocorre em crianças previamente sadias, com um pico entre 4 e 8 anos de idade. ${ }^{1,2}$

A PTI é uma doença imunomediada na qual as plaquetas são opsonizadas por autoanticorpos e destruídas pelos

\footnotetext{
- Belo Horizonte-MG.

Correspondência: Raquel Baumgratz Delgado

Serviço de Hematologia do Hospital das Clínicas da UFMG - Ambulatório Borges da Costa

Av. Alfredo Balena, 190

30130-100 - Belo Horizonte-MG - Brasil

Tel./Fax.: (+55 31) 3409-9397

Email: rgratz6262@yahoo.com.br
}

${ }^{1}$ Hematologista e hemoterapeuta do Serviço de Hematologia do Hospital das Clínicas da UFMG - Belo Horizonte-MG.

${ }^{2}$ Professor Titular do Depto. de Pediatria da UFMG. Coordenador do Serviço de Hematologia do Hospital das Clínicas da UFMG.

${ }^{3}$ Professora Assistente do Depto. de Pediatria da UFMG e membro do Serviço de Hematologia do Hospital das Clínicas da UFMG.

Serviço de Hematologia do Hospital das Clínicas da Universidade Federal de Minas Gerais (UFMG), Faculdade de Medicina da UFMG 
fagócitos do sistema reticuloendotelial. A trombocitopenia vista na PTI resulta do aumento do clearance plaquetário no baço e no fígado. Mais de $80 \%$ dos pacientes com PTI têm anticorpos reativos às glicoproteínas da superfície plaquetária, principalmente as glicoproteínas IIb-IIIa, Ia/IIa, ou Ib/IX. ${ }^{3}$

As plaquetas opsonizadas ligam-se às células apresentadoras de antígenos - macrófagos ou células dendríticas e são fagocitadas e destruídas. As células apresentadoras de antígenos amplificam a resposta imune inicial-determinant spreading - expressando novos peptídeos na sua superfície e iniciando a proliferação de clones de células T CD4-positivas. Os receptores de imunoglobulina das células B que reconhecem os antígenos plaquetários são também induzidos a proliferar e a sintetizar anticorpos antiglicoproteína. ${ }^{4}$

O diagnóstico da PTI recai na exclusão de outras causas de trombocitopenias. As chaves para o diagnóstico são a história e o exame clínico, o hemograma completo e uma análise do esfregaço de sangue periférico. ${ }^{5} \mathrm{~A}$ contagem plaquetária menor que $150 \times 10^{9} / \mathrm{L}$, associada a manifestações clínicas exclusivamente hemorrágicas, sem outras alterações do hemograma e do coagulograma, é o achado laboratorial essencial para o diagnóstico. ${ }^{6}$

A PTI aguda, de início súbito, ocorre principalmente na infância e, muitas vezes, sucede a uma infecção viral ou vacinação. A maioria das crianças afetadas não necessita tratamento medicamentoso, sendo que $80 \%$ a $85 \%$ dos casos têm resolução espontânea da doença dentro dos primeiros seis meses do diagnóstico. Cerca de 15\% a 20\% persistem trombocitopênicas por mais de seis meses - forma crônica - que se assemelha à doença nos adultos. A PTI crônica na criança tem uma incidência estimada de 0,46 por 100 mil crianças por ano. ${ }^{7}$ Estudos recentes tentam comprovar que mudanças no sistema imune ocorridas com o avançar da idade contribuem para as anormalidades observadas na PTI crônica. ${ }^{8}$

A criança com suspeita de PTI, quase invariavelmente trilha um de dois caminhos até chegar ao hematologista. Ela chega a uma unidade de Pronto Atendimento hospitalar apresentando as manifestações hemorrágicas da doença: epistaxe, gengivorragia, sangramento do trato gastrointestinal ou, raramente, hemorragia cerebral, cuja incidência tem sido estimada entre $0,1 \%$ a $1 \% .{ }^{9,10}$ Alternativamente, o paciente é encaminhado pelo pediatra, que observa petéquias e equimoses ou trombocitopenia no hemograma, durante consulta de rotina. O encaminhamento "plaquetopenia a esclarecer" é clássico no ambulatório de hematologia pediátrica.

A despeito da alta frequência da PTI na clínica hematológica pediátrica e do diagnóstico fácil e relativamente rápido, o seu manejo clínico ainda carece de consenso, especialmente nas questões que se referem a: realização do mielograma, hospitalização, tratamento e escolha da terapêutica inicial. ${ }^{11}$ As abordagens clínicas correntes, ainda que sob a tutela de protocolos internacionais, baseiam-se em experiências pessoais, estudos de casos não controlados ou ensaios clínicos randomizados com curto período de seguimento, nos quais a contagem plaquetária é a única variável considerada. ${ }^{12} \mathrm{~A}$ adesão a estes protocolos também é variável e, muitas vezes, a prática diverge completamente do estabelecido ou sugerido. ${ }^{13}$

Este estudo tem como objetivos caracterizar, de acordo com a experiência do Serviço de Hematologia do Hospital das Clínicas da UFMG, a história natural, a apresentação e o manejo clínico da PTI da criança; determinar a frequência dos quadros clínicos graves, moderados, leves e assintomáticos, e correlacioná-los com as respectivas contagens de plaquetas; determinar os fatores preditivos da resposta à esplenectomia, do desfecho - remissão ou atividade - e da evolução para a cronicidade.

\section{Casuística e Método}

Foram selecionados para revisão prontuários de pacientes de até 16 anos completos, com quadro clínico de púrpura, que foram submetidos ao mielograma no Serviço de Hematologia do Hospital das Clínicas da UFMG, no período de abril de 1988 a dezembro de 2001, e cujos resultados foram considerados "normais" ou descreviam apenas hiperplasia megacariocítica. Foram excluídos os resultados conclusivos para: calazar, leucemia aguda, anemia aplásica, lúpus eritematoso sistêmico e sorologia positiva para HIV.

Dados clínicos e laboratoriais de 187 pacientes foram coletados nos respectivos prontuários e o seguimento atualizado para 162 crianças até junho de 2004. Em 25 crianças, a atualização não foi possível, sendo utilizados os dados colhidos em estudo preliminar (Fernandes RAF et al., Tema livre, XVII Congresso do Colégio Brasileiro de Hematologia, 1999).

A título de uniformização das informações, foram adotados alguns conceitos nem sempre utilizados na literatura:

- Trombocitopenia ou PTI em atividade: plaquetas $<100 \times 10^{9} / \mathrm{L}$.

- PTI aguda: trombocitopenia com remissão dentro de seis meses.

- PTI crônica: trombocitopenia persistente por mais de seis meses.

- Remissão: duas contagens plaquetárias consecutivas com número de plaquetas $\geq 100 \times 10^{9} / \mathrm{L}$.

- Data da remissão: a data correspondente à primeira de duas contagens consecutivas de plaquetas $\geq 100 \times 10^{9} / \mathrm{L}$.

- Remissão espontânea: remissão estando o paciente sem uso de medicamentos há pelo menos seis meses.

- Recaída: todo episódio de queda do número de plaquetas $<100 \times 10^{9} / \mathrm{L}$.

- O início da PTI foi considerado súbito quando as manifestações clínicas surgiram até duas semanas antes do diagnóstico; insidioso, quando esse período foi superior a duas semanas. 
- Quanto à forma de apresentação clínica, ignorando a contagem de plaquetas e tomando emprestados os conceitos sugeridos por Bolton-Maggs e Moon ${ }^{13}$, a PTI foi classificada em:

1. Assintomática: ausência de manifestações clínicas;

2. Leve: hematomas e petéquias, epistaxe discreta e ocasional, pequena ou nenhuma interferência na vida diária;

3. Moderada: manifestações cutâneas mais graves com algumas lesões de mucosa, epistaxe ou menorragia de manejo mais difícil;

4. Grave: episódios hemorrágicos, epistaxe, menorragia e/ou melena, requerendo internação hospitalar e/ou transfusão sanguínea; manifestações hemorrágicas que afetam seriamente a qualidade de vida do paciente.

A possível associação entre variáveis nominais foi verificada pelo teste do qui-quadrado, com a correção de Yates. Quando o valor esperado numa das caselas de tabela de contingência foi menor que 5, utilizou-se o teste exato de Fisher. Quando uma das variáveis nominais foi estratificada em três ou mais categorias foi utilizado o teste do qui-quadrado de tendência para verificar a associação com outra variável nominal.

A distribuição não-normal de valores de uma variável contínua foi comparada utilizando-se o teste de KruskalWallis. Sempre que a variável contínua apresentou distribuição normal, as médias foram comparadas com o teste $t$ de Student.

As crianças que se encontravam com doença em atividade e seguimento inferior a seis meses, na data da última contagem de plaquetas, foram excluídas da análise estatística de associação entre o desfecho clínico e outras variáveis clínicas e laboratoriais. Em todos os testes estatísticos, utilizou-se a probabilidade de erro alfa $\mathrm{p} \leq 0,05$ para se descartar a hipótese nula subjacente ao teste empregado. A pesquisa foi aprovada pelo Comitê de Ética em Pesquisa da UFMG (Parecer 142/02).

\section{Resultados}

Foram pesquisados prontuários de 187 pacientes, sendo 59,4\% meninas. As idades variaram de 2 meses a 15,7 anos (mediana de 6,2 anos).

História de infecção prévia ao diagnóstico da PTI foi relatada em um terço dos casos.

Manifestações hemorrágicas foram descritas em 177 casos (94,7\%). Oito pacientes encontravam-se sem sinais ou sintomas, sendo o diagnóstico baseado no achado casual de plaquetopenia em hemograma. Essa informação não estava registrada em dois prontuários. Dos 177 pacientes, 103 (58,2\%) apresentaram apenas um tipo de manifestação hemorrágica: 99 somente manifestações cutâneas (petéquias e equimoses) e quatro apenas hemorragias mucosas (epistaxe e gengivorragia). Os demais 74 casos sintomáticos apresen- taram a associação de duas ou mais manifestações hemorrágicas. A associação mais frequente foi a de manifestações hemorrágicas cutâneas e epistaxe, em 25 casos (33,8\%). Hemorragia do trato gastrointestinal ocorreu em 16 pacientes e do trato genitourinário em 15 , ambas sempre associadas a outras manifestações hemorrágicas. Apenas um paciente apresentou hemorragia intracraniana e esta foi consequência de traumatismo craniano.

O exame físico não detectou alterações além das manifestações hemorrágicas em 151 pacientes. As alterações detectadas nos 36 pacientes restantes foram: linfadenomegalia, hepatomegalia, hepatoesplenomegalia e alterações diversas como sinais de choque hipovolêmico, dispnéia, prostração, tiragem intercostal e alterações dismórficas.

Quanto à gravidade das manifestações hemorrágicas, 9,2\% foram classificados como graves, $15,1 \%$ como moderados, 71,4\% como leves e 4,3\% eram assintomáticos.

O número de plaquetas ao diagnóstico estava registrado em 182 prontuários e variou de 0 a $96 \times 10^{9} / \mathrm{L}$, com mediana de $13 \times 10^{9} / \mathrm{L}$ (Figura 1 ).

A relação entre gravidade dos sintomas hemorrágicos e a contagem de plaquetas à apresentação inicial da PTI é ilustrada na Figura 2.

A PTI se iniciou de forma súbita em 134 pacientes (71,7\%) e de forma insidiosa em 49 (26,2\%), Tabela 1 . O início da doença não foi especificado em quatro prontuários.

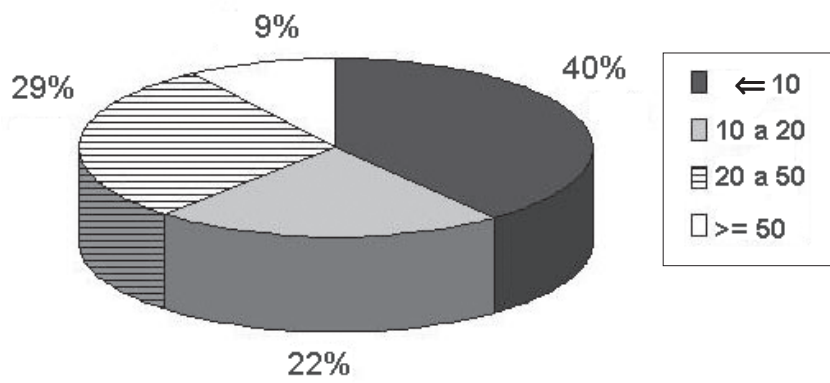

Figura 1. Número de plaquetas ao diagnóstico (x10\%/L) em 182 crianças com Púrpura Trombocitopênica Imune

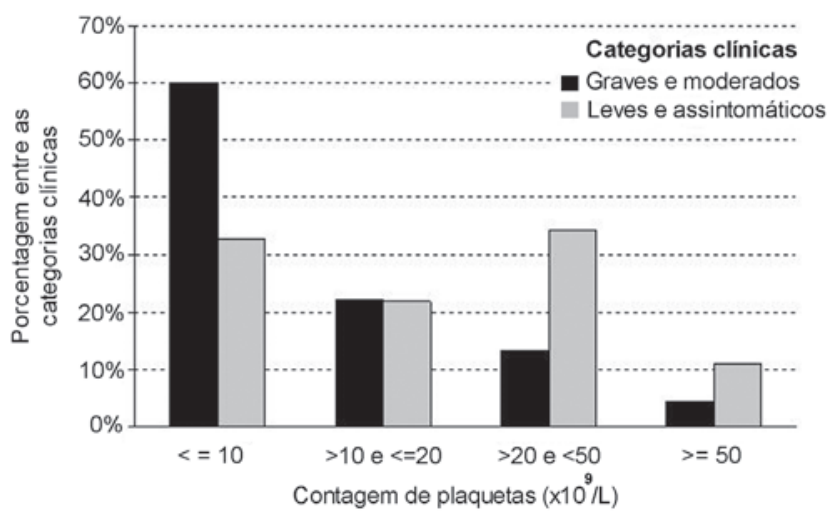

Figura 2. Gravidade clínica do sangramento, segundo BoltonMaggs $^{13}$, e contagem de plaquetas ao diagnóstico em 182 crianças com Púrpura Trombocitopênica Imune 
Tabela 1. Características da PTI quanto à forma de apresentação

\begin{tabular}{|c|c|c|c|}
\hline & $\begin{array}{l}\text { Apresentação } \\
\text { súbita }\end{array}$ & $\begin{array}{l}\text { Apresentação } \\
\text { insidiosa }\end{array}$ & $\begin{array}{l}\text { Valores } \\
\text { de } p\end{array}$ \\
\hline Número de pacientes & 134 & 49 & \\
\hline $\begin{array}{l}\text { Tempo de seguimento } \\
\text { (meses) }\end{array}$ & $\begin{array}{c}0,2 \text { a } 135,2 \\
\text { (mediana } 11,8)\end{array}$ & $\begin{array}{c}0,9 \text { a } 145,3 \\
\text { (mediana } 43,1 \text { ) }\end{array}$ & \\
\hline Sexo (masc/fem) & $61(45,5 \%) / 73(54,5 \%$ & $13(26,5 \%) / 36(73,5 \%)$ & 0,003 \\
\hline $\begin{aligned} & \text { Idade (anos) } 0 \text { a } 4 \\
& 4 \text { a } 8 \\
& 8 \text { a } 10 \\
&>10\end{aligned}$ & $\begin{array}{c}54 / 60(90 \%) \\
39 / 57(68,4 \%) \\
13 / 23(56,5 \%) \\
28 / 43(65,1 \%)\end{array}$ & $\begin{array}{c}6 / 60(10 \%) \\
18 / 57(31,6 \%) \\
10 / 23(43,5 \%) \\
15 / 43(34,9 \%)\end{array}$ & 0,003 \\
\hline $\begin{array}{l}\text { Manifestações clínicas: } \\
\text { Assintomáticos } \\
\text { Leves } \\
\text { Moderados } \\
\text { Graves }\end{array}$ & $\begin{array}{c}4(3 \%) \\
95(71 \%) \\
20(15 \%) \\
14(11 \%)\end{array}$ & $\begin{array}{c}3(6 \%) \\
36(74 \%) \\
7(14 \%) \\
3(6 \%)\end{array}$ & n.s. \\
\hline $\begin{array}{l}\text { Esplenectomia } \\
\text { Resposta à esplenectomia }\end{array}$ & $\begin{array}{l}20(15 \%) \\
11(55 \%)\end{array}$ & $\begin{array}{c}26(53 \%) \\
11(42,3 \%)\end{array}$ & $\begin{array}{c}4 \times 10^{-7} \\
\text { n.s. }\end{array}$ \\
\hline Corticoterapia & $109(81,3 \%)$ & $40(81,6 \%)$ & n.s. \\
\hline Resposta à corticoterapia & $77(70,6 \%)$ & $17(42,5 \%)$ & 0,003 \\
\hline Recaídas & $28(21 \%)$ & $18(37 \%)$ & 0,05 \\
\hline $\begin{array}{l}\text { Curso clínico* } \\
\text { Agudo } \\
\text { Crônico }\end{array}$ & $\begin{array}{c}102(76,1 \%) \\
21(15,7 \%)\end{array}$ & $\begin{array}{c}19(38,8 \%) \\
29(59 \%)\end{array}$ & $1 \times 10^{-7}$ \\
\hline $\begin{array}{l}\text { Desfecho clínico final } \\
\text { Remissão } \\
\text { Atividade } \dagger \\
\text { Plaq }>100 \times 10 \% \text {, sem } \\
\text { critério de remissão } \\
\text { Óbito }\end{array}$ & $\begin{array}{c}111(82,8 \%) \\
6(4,5 \%) \\
10(7,5 \%) \\
1(0,8 \%)\end{array}$ & $\begin{array}{c}36(73,5 \%) \\
9(18,4 \%) \\
4(8,1 \%) \\
0\end{array}$ & 0,006 \\
\hline
\end{tabular}

n.s.: não significativo

* Treze pacientes excluídos devido ao tempo de seguimento inferior a seis meses

$\dagger$ Seis pacientes excluídos devido ao tempo de seguimento inferior a seis meses

esplenectomizados daqueles nos quais ela não foi indicada. A apresentação insidiosa foi significativamente mais frequente nos que necessitaram de esplenectomia (Tabela 1) e naqueles classificados como portadores de PTI crônica (Tabela 2). A idade à esplenectomia variou de 1,2 a 18,7 anos (mediana de 9,7 anos). Oito pacientes (17\%) foram esplenectomizados antes de completarem 5 anos de idade.

O tempo de seguimento após a esplenectomia variou de 2 dias a 3 anos, com mediana de 1 ano e 9 meses. Ao término do seguimento, 35 pacientes (74,5\% dos esplenectomizados) estavam em remissão, seis (12,8\%) com doença em atividade, cinco (10,6\%) possuíam uma única plaquetometria $>100 \times 10^{9} / \mathrm{L}$ e, portanto, sem critério para remissão e ocorreu um óbito (2,1\%). Dos 35 pacientes esplenectomizados que atingiram a remissão, 33 não necessitaram de terapêutica adicional posterior. A contagem de plaquetas $\geq 300 \times 10^{9} / \mathrm{L}$, no intervalo de 7 a 99 dias pós-esplenectomia, constituiu-se em fator preditivo para a remissão de 18 pacientes, quando comparados aos seis pacientes nos quais a doença encontrava-se em atividade $(p=0,006)$.

O tempo de seguimento para as crianças que foram consideradas em remissão variou de 6 dias a 12 anos (mediana de 1 ano e 5 meses). Para as crianças com doença em atividade, esse tempo variou de 6 dias a 9 anos e 3 meses (mediana de 2 anos).

O curso clínico foi estudado em 174 pacientes. Os dados de 13 pacientes foram excluídos dessa análise devido a abandono do seguimento. Evoluíram como PTI aguda 123 pacientes (70,7\% dos casos analisados) e, como PTI crônica, 51 (29,3\%) (Tabela 2).

Não receberam qualquer tratamento medicamentoso ao diagnóstico 34 pacientes do total de casos do estudo (18,2\%). Corticóide foi o tratamento inicial para 81,3\% dos pacientes. Nenhum paciente recebeu imunoglobulina venosa (IgIV) como terapia inicial. Do total de 38 pacientes que usaram o medicamento, sete o receberam como preparo para a esplenectomia. Resposta traduzida como primeira remissão após o uso de imunoglobulina e não associada à esplenectomia foi observada em seis pacientes (15,8\%).

Dos 187 pacientes do estudo, 47 foram esplenectomizados (25,1\%), 19 meninos e 28 meninas. Nenhum paciente foi submetido à esplenectomia como tratamento inicial. A gravidade das manifestações hemorrágicas, ou a plaquetometria inicial, não distinguiu os pacientes que, ulteriormente, foram
Remissão foi a situação final para 150 dos 187 pacientes estudados (80,2\%). Outros 15 pacientes (8\%) apresentavam número de plaquetas maior ou igual a $100 \times 10^{9} / \mathrm{L}$, ao término do seguimento, sem contudo preencherem o critério de remissão.

Vinte e um pacientes (11,2\% do total do estudo) estavam com a doença em atividade, ao término do seguimento, mas seis foram seguidos por tempo inferior a 6 meses e foram excluídos da análise estatística (Tabela 3).

O número de plaquetas ao diagnóstico variou de $8 \times 10^{9} / \mathrm{L}$ a $75 \times 10^{9} / \mathrm{L}$, com mediana de $27 \times 10^{9} / \mathrm{L}$. A Figura 3 ilustra a relação entre a plaquetometria ao diagnóstico e a situação dos pacientes ao final do estudo.

\section{Discussão}

A proporção encontrada entre meninas e meninos acometidos de 1,4:1 e a idade pico para o diagnóstico entre 2 e 6 anos de idade confirmam os dados clássicos da literatura, ${ }^{14}$ 
Tabela 2. Características clínicas e terapêuticas da PTI aguda e crônica

\begin{tabular}{|c|c|c|c|}
\hline & PTI aguda & PTI crônica & $\begin{array}{l}\text { Valores } \\
\text { de } p\end{array}$ \\
\hline Número de pacientes & $123(70,7 \%)$ & $51(29,3 \%)$ & \\
\hline $\begin{array}{l}\text { Tempo de seguimento } \\
\text { (em meses) }\end{array}$ & $\begin{array}{c}0,2 \text { a } 145,3 \\
\text { (mediana: } 12,5 \text { ) }\end{array}$ & $\begin{array}{c}\text { 6,2 a } 111 \\
\text { (mediana: } 34,8 \text { ) }\end{array}$ & \\
\hline Sexo (masc/fem) & $53(43 \%) / 70(57 \%)$ & $15(29 \%) / 36(71 \%)$ & n.s. \\
\hline $\begin{aligned} \text { Idade (anos) } & 0 \text { a } 4 \\
& 4 \text { a } 8 \\
& 8 \text { a } 10 \\
& >10\end{aligned}$ & $\begin{array}{c}44 / 56(78,6 \%) \\
41 / 53(77,4 \%) \\
13 / 22(59 \%) \\
25 / 43(58,1 \%)\end{array}$ & $\begin{array}{c}12 / 56(21,4 \%) \\
12 / 53(22,6 \%) \\
9 / 22(41 \%) \\
18 / 43(41,9 \%)\end{array}$ & 0,057 \\
\hline $\begin{array}{l}\text { Infecção prévia relatada } \\
\text { Afirmada } \\
\text { Negada } \\
\text { Não relatada }\end{array}$ & $\begin{array}{c}115(93,5 \%) \\
45(39,1 \%) \\
70(60,9 \%) \\
8\end{array}$ & $\begin{array}{c}45(88,2 \%) \\
11(24,4 \%) \\
34(75,6 \%) \\
6\end{array}$ & n.s. \\
\hline $\begin{array}{l}\text { Uso prévio de drogas relatado: } \\
\text { Afirmado } \\
\text { Negada } \\
\text { Não relatada }\end{array}$ & $\begin{array}{c}118(96 \%) \\
25(21,2 \%) \\
93(78,8 \%) \\
5\end{array}$ & $\begin{array}{c}48(94 \%) \\
6(12,5 \%) \\
42(87,5 \%) \\
3\end{array}$ & n.s. \\
\hline $\begin{array}{l}\text { Forma de apresentação relatada: } \\
\text { Súbita } \\
\text { Insidiosa } \\
\text { Não relatada }\end{array}$ & $\begin{array}{c}121(98,4 \%) \\
102(84,3 \%) \\
19(15,7 \%) \\
2\end{array}$ & $\begin{array}{c}50(98 \%) \\
21(42 \%) \\
29(58 \%) \\
1\end{array}$ & $1 \times 10^{-7}$ \\
\hline 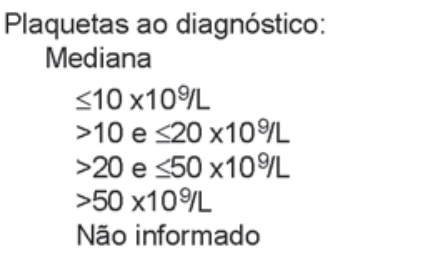 & $\begin{array}{c}12 \times 10 \% / L \\
53(44,2 \%) \\
24(20 \%) \\
33(27,5 \%) \\
10(8,3 \%) \\
3\end{array}$ & $\begin{array}{c}13 \times 10 \% / \mathrm{L} \\
17(34 \%) \\
14(28 \%) \\
16(32 \%) \\
3(6 \%) \\
1\end{array}$ & n.s. \\
\hline $\begin{array}{l}\text { Esplenectomia } \\
\text { Resposta à esplenectomia }\end{array}$ & $\begin{array}{l}18(14,6 \%) \\
5(27,8 \%)\end{array}$ & $\begin{array}{l}29(56,9 \%) \\
18(62,1 \%\end{array}$ & $\begin{array}{l}<1 \times 10^{-7} \\
0,047\end{array}$ \\
\hline $\begin{array}{l}\text { Tratamento inicial } \\
\text { Corticoterapia } \\
\text { Resposta à corticoterapia }\end{array}$ & $\begin{array}{l}104(84,5 \%) \\
89(85,5 \%)\end{array}$ & $\begin{array}{c}47(74,6 \%) \\
5(10,6 \%)\end{array}$ & $\begin{array}{c}\text { n.s. } \\
<1 \times 10^{-7}\end{array}$ \\
\hline $\begin{array}{l}\text { Desfecho clínico final } \\
\text { Remissão } \\
\text { Atividade } \\
\text { Plaq } 100 \times 10 \% / \mathrm{L} \text { sem } \\
\text { critério de remissão } \\
\text { Óbito }\end{array}$ & $\begin{array}{c}117(95,1 \%) \\
3(2,4 \%) \\
2 \\
1\end{array}$ & $\begin{array}{l}33(64,7 \%) \\
12(23,5 \%) \\
6\end{array}$ & $1 \times 10^{-5}$ \\
\hline
\end{tabular}

n.s.: não significativo

* Seis pacientes excluídos devido ao tempo de seguimento inferior a seis meses.
Além disso, vários casos típicos de PTI aguda podem não ter sido submetidos à realização do mielograma, o que os colocou fora do contexto deste estudo. Dos 187 pacientes estudados, 74,9\% apresentaram a primeira remissão dentro de 12 meses do diagnóstico, próximo aos $80 \%$ relatados na literatura. ${ }^{17}$ Constituíram-se fatores associados significativamente à remissão final o gênero masculino, o número mais baixo de plaquetas ao diagnósti$\mathrm{co}$, a resposta à corticoterapia inicial e a ocorrência de uma primeira remissão, constatação semelhante à de outros autores. ${ }^{16,18,19}$

A classificação clínica sugerida por Bolton-Maggs e Moon ${ }^{13}$ foi adotada com o objetivo semelhante de fornecer dados adicionais além da contagem plaquetária, para caracterização da gravidade dos quadros hemorrágicos. Assim como relatado por esses autores, a maioria dos pacientes apresentou apenas sintomas cutâneos e os quadros leves foram os mais comumente observados $-74 \%$ nos dados dos autores citados e 71,4\% na casuística estudada.

A correlação entre número de plaquetas à apresentação e gravidade dos sintomas não repetiu a observação feita pelos mesmos autores de que $70 \%$ (181/ 260) das crianças com plaquetas abaixo de $10 \times 10^{9} / \mathrm{L}$ apresentavam quadro clínico leve. ${ }^{13} \mathrm{Na}$ presente casuística, entre as crianças com esta contagem, cerca de 60\% (44/73) apresentavam quadro clínico moderado ou grave. Utilizando sistemas diferentes de classificação, outros autores encontraram resultados semelhantes ao do presente estudo e demonstraram uma tendência do grau de sangramento aumentar à medida que o número de plaquetas diminui: 97\% dos episódios hemorrágicos graves e moderados ocorreram quando o número de plaquetas era $<20 \times 10^{9} / \mathrm{L}^{20,21}$ No atual estudo, cerca de $80 \%$ dos casos graves e moderados apresentaram essas mesmas contagens plaquetárias. Recentemente, foi descrito um método para quantificação objetiva dos sintomas hemorrágicos da PTI: o IBLS - ITP Bleeding Scale, considerando casos de PTI aguda e crônica em adultos e crianças e a contagem total de plaquetas e de plaquetas gigantes. ${ }^{22} \mathrm{O}$ estudo demonstrou que, na vigência de contagem plaquetária abaixo de $30 \times 10^{9} / \mathrm{L}$, não existe correlação significativa entre os dados laboratoriais e a 
Tabela 3. Características da PTI quanto ao desfecho clínico

\begin{tabular}{|c|c|c|c|}
\hline & Atividade & Remissão & $\begin{array}{l}\text { Valores } \\
\text { de } p\end{array}$ \\
\hline Número de pacientes & $15(8 \%)$ & $150(80,2 \%)$ & \\
\hline Sexo (masc/fem) & $1 / 14$ & $62 / 88$ & 0,02 \\
\hline $\begin{aligned} & \text { Idade (anos) } 0 \text { a } 4 \\
& 4 \text { a } 8 \\
& 8 \text { a } 10 \\
&>10\end{aligned}$ & $\begin{array}{c}4 / 54(7,4 \%) \\
5 / 49(10,2 \%) \\
3 / 22(13,6 \%) \\
3 / 40(7,5 \%)\end{array}$ & $\begin{array}{l}50 / 54(92,6 \%) \\
44 / 49(89,8 \%) \\
19 / 22(86,4 \%) \\
37 / 40(92,5 \%)\end{array}$ & n.s. \\
\hline $\begin{array}{l}\text { Infecção prévia relatada: } \\
\text { Afirmada } \\
\text { Negada } \\
\text { Não relatada }\end{array}$ & $\begin{array}{c}2(13,3 \%) \\
13(86,6 \%) \\
0\end{array}$ & $\begin{array}{c}52(34,7 \%) \\
84(56 \%) \\
14\end{array}$ & n.s. \\
\hline $\begin{array}{l}\text { Uso prévio de drogas relatado } \\
\text { Afirmado } \\
\text { Negado } \\
\text { Não relatado }\end{array}$ & $\begin{array}{c}3(20 \%) \\
12(80 \%) \\
0\end{array}$ & $\begin{array}{c}28(18,7 \%) \\
114(76 \%) \\
8\end{array}$ & n.s. \\
\hline $\begin{array}{l}\text { Forma de apresentação relata } \\
\text { Súbita } \\
\text { Insidiosa } \\
\text { Não relatada }\end{array}$ & $\begin{array}{c}6(40 \%) \\
9(60 \%) \\
0\end{array}$ & $\begin{array}{c}111(74 \%) \\
36(24 \%) \\
3\end{array}$ & 0,006 \\
\hline $\begin{array}{l}\text { Quadro clínico descrito } \\
\text { Leve } \\
\text { Moderado } \\
\text { Grave } \\
\text { Assintomático } \\
\text { Não informado }\end{array}$ & $\begin{array}{c}15(100 \%) \\
10(66,7 \%) \\
2(13,3 \%) \\
2(13,3 \%) \\
1(6,7 \%) \\
0\end{array}$ & $\begin{array}{c}146(97,3 \%) \\
108(74,0 \%) \\
24(16,4 \%) \\
14(9,6 \%) \\
3 \\
1\end{array}$ & n.s \\
\hline $\begin{array}{l}\text { Plaquetas ao diagnóstico } \\
\text { Mediana } \\
\leq 10 \times 10^{9} / \mathrm{L} \\
>10 \text { e } \leq 20 \times 10^{9} / \mathrm{L} \\
>20 \text { e } \leq 50 \times 10^{9} / \mathrm{L} \\
>50 \times 10^{9} / \mathrm{L} \\
\text { Não informado }\end{array}$ & $\begin{array}{c}27 \times 10 \% / L \\
1(6,7 \%) \\
4(26,7 \%) \\
8(53,3 \%) \\
2(13,3 \%) \\
0\end{array}$ & $\begin{array}{c}12 \times 10^{9} / \mathrm{L} \\
66(45,2 \%) \\
34(23,3 \%) \\
36(24,7 \%) \\
10(6,8 \%) \\
4\end{array}$ & 0,004 \\
\hline $\begin{array}{l}\text { Esplenectomia } \\
\text { Resposta à esplenectomia }\end{array}$ & $\begin{array}{c}6(40 \%) \\
0\end{array}$ & $\begin{array}{l}35(23,3 \%) \\
21(60 \%)\end{array}$ & $\begin{array}{c}\text { n.s } \\
0,009\end{array}$ \\
\hline $\begin{array}{l}\text { Tratamento inicial } \\
\text { Corticoterapia } \\
\text { Resposta à corticoterapia } \\
\text { Primeira remissão }\end{array}$ & $\begin{array}{c}12(80 \%) \\
3(25 \%) \\
4(26,7 \%)\end{array}$ & $\begin{array}{l}128(85,3 \%) \\
89(69,5 \%) \\
150(100 \%)\end{array}$ & $\begin{array}{c}\text { n.s. } \\
0,003 \\
<1 \times 10^{-7}\end{array}$ \\
\hline
\end{tabular}

n.s.: não significativo

gravidade do sangramento. Em outro estudo ${ }^{23}$ observou-se que a frequência de manifestações hemorrágicas leves era maior nos episódios em que a contagem de plaquetas situava-se entre 10 e 20x10\%/L, quando comparada com episódios com contagens inferiores a $10 \times 10^{9} / \mathrm{L}$. Todos os estudos, inclusive o aqui apresentado, trazem as limitações advindas da dificuldade em se definir a medida da hemorragia, seja cutânea ou mucosa (wet purpura, dry purpura), e da superposição que muitas vezes ocorre entre um grau e outro na classificação.

A PTI se apresentou de forma súbita em 134 pacientes e de forma insidiosa em 49, números semelhantes aos descritos em estudo clássico, ${ }^{24}$ no qual 181 crianças com PTI foram avaliadas ( 132 formas agudas e 45 formas insidiosas). No presente estudo, a PTI nas meninas com mais idade associou-se a uma tendência significativa para a apresentação insidiosa ( $\mathrm{p}=0,03$ e 0,003 , respectivamente para sexo e idade). Também foi no grupo de apresentação insidiosa que esplenectomias foram mais realizadas. Esses dados relacionam-se com a observação de que houve uma associação estatisticamente significativa entre forma insidiosa e PTI crônica e forma de apresentação súbita e PTI de evolução aguda $\left(\mathrm{p}=1 \times 10^{-7}\right)$. Estudo feito em crianças canadenses confirmou essas observações..$^{18} \mathrm{~A}$ apresentação insidiosa foi a única variável apontada como fator preditivo da cronicidade ( $p=0,001)$, em outro relato. ${ }^{25}$ Estudo feito em países nórdicos chegou a conclusão semelhante. ${ }^{16}$

Outra associação relevante foi a menor resposta à corticoterapia no grupo de início insidioso $(p=0,003)$, dado equivalente ao observado quando da comparação da mesma resposta entre os pacientes com PTI aguda e os com PTI crôni$\mathrm{ca}\left(\mathrm{p}<1 \times 10^{-7}\right)$. Contudo, a maioria dos autores concorda que nenhuma terapia parece mudar a história natural da doença.

A esplenectomia foi realizada em $25 \%$ dos casos e a contagem plaquetária, mais do que a sintomatologia, parece ter sido o parâmetro determinante para sua realização. Os principais guidelines internacionais ${ }^{1,11,26}$ estabelecem critérios diferentes para a indicação da realização da esplenectomia na PTI da criança. A tendência atual tem sido mais conservadora, tanto no sentido de postergar ao máximo a sua realização, quanto no sentido de tentar delimitar fatores preditivos da resposta à cirurgia. No presente estudo, a contagem de plaquetas $\geq 300 \times 10^{9} / \mathrm{L}$, no intervalo de 7 a 99 dias após a esplenectomia, constituiu-se em fator preditivo para a remissão. Alguns autores têm demonstrado que uma boa resposta à IgIV parece ser preditiva de uma boa resposta à esplenectomia, mas uma não-resposta à IgIV não pode definir o fracasso ou o sucesso da cirurgia. $27-29$

A taxa de resposta à esplenectomia, de $74,5 \%$, num tempo mediano de seguimento de 1 ano e 9 meses, aproximou-se dos resultados de outros autore ${ }^{30}$ que encontraram taxa de resposta de 76,3\%, num seguimento médio de 2,1 anos.

O pequeno número de pacientes que não receberam qualquer tipo de tratamento, 34 (18,2\%), contrasta com o alto número de pacientes assintomáticos e com manifestações clínicas leves (140 casos do total de 185 pacientes). Considerando que 153 pacientes (81,8\%) receberam algum 


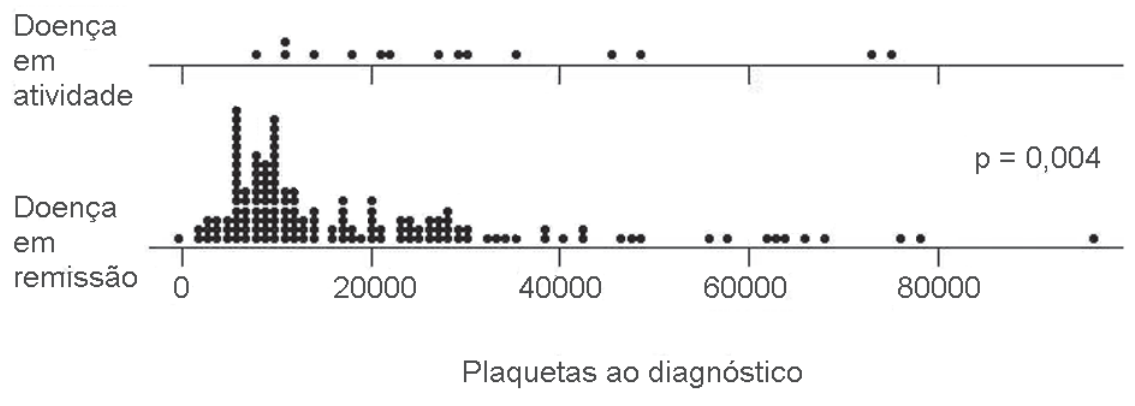

Figura 3. Número de plaquetas ao diagnóstico e desfecho clínico ao final do estudo em 161 crianças com Púrpura Trombocitopênica Imune.

tipo de tratamento e que $62 \%$ tinham plaquetas abaixo de 20x10\%/L ao diagnóstico, pode-se inferir que a contagem plaquetária, mais do que os sintomas, foi o principal parâmetro na decisão da conduta terapêutica. A avaliação da adesão de médicos pediatras e hematologistas pediátricos ao protocolo britânico de tratamento da PTI de $19922^{11}$ demonstrou que $60 \%$ dos pacientes com PTI foram tratados com o objetivo de elevar o número de plaquetas, apesar da maioria (74\%) ter sido classificada como clinicamente leve. ${ }^{13} \mathrm{Um}$ pequeno estudo com 55 crianças recém-diagnosticadas com $\mathrm{PTI}^{31}$ demonstrou que a conduta expectante, sem qualquer medicamento, foi adequada, mesmo quando a plaquetometria era inferior a $10 \times 10^{9} / \mathrm{L}$ ( $67 \%$ dos casos).

Apenas 35 pacientes, do total de 152 que receberam corticóide como terapia inicial, usaram o medicamento no limite de duração preconizado pelos guidelines mais divulgados. ${ }^{1,11,26}$ Cento e dezessete pacientes (77\%) ultrapassaram os 21 dias de uso, e as taxas de resposta foram semelhantes para ambos os grupos, indicando não haver benefício na manutenção do medicamento por longo prazo.

A despeito do recomendado pela American Society of Hematology ${ }^{1}$, nenhum paciente recebeu IgIV como primeira terapia. $\mathrm{O}$ alto custo do medicamento foi, seguramente, o principal fator limitante de sua indicação.

A hemorragia intracraniana ocorreu em apenas um paciente $(0,5 \%)$ confirmando que, ainda que grave, ela é uma complicação rara. Esse número não pode ser levado à comparação com os dados da literatura, por não considerar os dados de incidência da PTI infantil.

Em conclusão, o presente estudo observou que a apresentação clínica da PTI da criança atendida no HC-UFMG foi semelhante à descrita nos estudos nacionais e internacionais, no que diz respeito à idade pico para o diagnóstico, à frequência entre os sexos, às manifestações clínico-laboratoriais e à evolução aguda ou crônica. As manifestações hemorrágicas exclusivamente cutâneas em 96\% dos casos sintomáticos, os quadros leves e assintomáticos apresentados por $76 \%$ dos pacientes e a taxa de remissão final de 95,1\% confirmaram que a PTI aguda da criança é essencialmente benigna e autolimitada. Constatou-se uma correlação entre gravidade dos sintomas e intensidade da plaquetopenia.
Pacientes do sexo feminino e com mais idade apresentaram uma tendência significativa para a forma insidiosa e esta associou-se a um maior número de esplenectomias e a uma menor taxa de resposta à corticoterapia. Constituíram-se fatores preditivos da cronicidade a apresentação insidiosa e a ausência de resposta à corticoterapia. Remissão foi a situação final para $80,2 \%$ dos pacientes. Constituíram-se fatores associados significativamente à remissão final o gênero masculino, o número mais baixo de plaquetas ao diagnóstico, a resposta à corticoterapia inicial e a ocorrência de uma primeira remissão. A esplenectomia foi realizada em $25 \%$ dos casos com taxa de resposta de $74,5 \%$.

\section{Abstract}

The objective of this work was to determine the frequency of clinical manifestations of immune thrombocytopenic purpura and its association with platelet count, response to splenectomy and predictive factors for response and chronicity. This retrospective study included 187 children diagnosed at Hospital das Clínicas, Federal University of Minas Gerais, Brazil, between April 1988 and December 2001. About 76\% of the cases had asymptomatic or clinically mild disease. Cutaneous bleeding alone was seen in $96 \%$ of the symptomatic cases. Severity of symptoms was associated with low platelet count. The acute disease was observed in 123 patients (70.7\%) and 51 (29.3\%) developed the chronic disease. Insidious presentation (26.2\%) was associated with a larger number of splenectomies $\left(p=4 \times 10^{-7}\right)$ and with a reduced response to steroids $(p=0.003)$. Additionally, the lack of response to steroids $\left(p<1 \times 10^{-7}\right)$ and insidious presentation were predictive factors for chronicity $\left(p=1 \times 10^{-7}\right)$. Splenectomy resulted in remission in $74.5 \%$. Remission was the final outcome for $80.2 \%$ of patients. Remission-associated factors included being male $(p=0.02)$, a lower platelet count at diagnosis $(p=0.004)$, response to steroids $(p=0.003)$, and the occurrence of a first remission $\left(p<1 \times 10^{-7}\right)$. The disease in childhood is benign and self-limiting with severity of symptoms being associated with a low platelet count. Rev. Bras. Hematol. Hemoter. 2009;31(1):29-36.

Key words: Immune thrombocytopenic purpura; idiopathic thrombocytopenic purpura; thrombocytopenia; child.

\section{Referências Bibliográficas}

1. George JN, Woolf SH, Raskob GE, Wasser JS, Aledort LM, Ballem PJ, et al. Idiopathic thrombocytopenic purpura: a practice guideline developed by explicit methods for the American Society of Hematology. Blood. 1996;88(1):3-40.

2. Medeiros D, Buchanan GR. Idiopathic thrombocytopenic purpura: beyond consensus. Curr Opin Pediatr. 2000;12(1):4-9.

3. Beardsley DS. ITP in the 21st century. Hematology Am Soc Hematol Educ Program. 2006:402-7. 
4. Cines DB, Blanchette VS. Immune thrombocytopenic purpura. N Engl J Med. 2002;346(13):995-1008.

5. Geddis AE, Balduini CL. Diagnosis of immune thrombocytopenic purpura in children. Curr Opin Hematol. 2007;14(5):520-5.

6. Thachil J, Hall GW. Is this immune thrombocytopenic purpura? Arch Dis Child. 2008;93(1):76-81.

7. Reid MM. Chronic idiopathic thrombocytopenic purpura: incidence, treatment, and outcome. Arch Dis Child. 1995;72 (2):125-8.

8. Culic S, Labar B, Marusic A, Salamunic I. Correlations among age, cytokines, lymphocyte subtypes, and platelet counts in autoimmune thrombocytopenic purpura. Pediatr Blood Cancer. 2006;47(5 Suppl):671-4.

9. Iyori H, Bessho F, Ookawa H, Konishi S, Shirahata A, Miyazaki S, et al. Intracranial hemorrhage in children with immune thrombocytopenic purpura. Japanese Study Group on childhood ITP. Ann Hematol. 2000;79(12):691-5.

10. Lilleyman JS. Intracranial haemorrhage in idiopathic thrombocytopenic purpura. Paediatric Haematology Forum of the British Society for Haematology. Arch Dis Child. 1994; 71(3):251-3.

11. Eden OB, Lilleyman JS. Guidelines for management of idiopathic thrombocytopenic purpura. The British Paediatric Haematology Group. Arch Dis Child. 1992;67(8):1056-8.

12. Vesely SK, Buchanan GR, Adix L, George JN, Cohen AR, Blanchette VS, et al. Self-reported initial management of childhood idiopathic thrombocytopenic purpura: results of a survey of members of the American Society of Pediatric Hematology/Oncology, 2001. J Pediatr Hematol Oncol. 2003;25(2):130-3.

13. Bolton-Maggs PH, Moon I. Assessment of UK practice for management of acute childhood idiopathic thrombocytopenic purpura against published guidelines. Lancet. 1997;350(9078): 620-3.

14. Blanchette V, Carcao M. Approach to the investigation and management of immune thrombocytopenic purpura in children. Semin Hematol. 2000;37(3):299-314.

15. Fogarty PF, Segal JB. The epidemiology of immune thrombocytopenic purpura. Curr Opin Hematol. 2007;14(5):515-9.

16. Zeller B, Rajantie J, Hedlund-Treutiger I, Tedgård U, Wesenberg F, Jonsson OG, et al. Childhood idiopathic thrombocytopenic purpura in the Nordic countries: epidemiology and predictors of chronic disease. Acta Paediatr. 2005;94(2):178-84.

17. Medeiros D, Buchanan GR. Major hemorrhage in children with idiopathic thrombocytopenic purpura: immediate response to therapy and long-term outcome. J Pediatr. 1998;133(3):334-9.

18. Belletrutti M, Ali K, Barnard D, Blanchette V, Chan A, David M, et al. Chronic immune thrombocytopenic purpura in children: a survey of the canadian experience. J Pediatr Hematol Oncol. 2007; 29(2):95-100.

19. Kühne T, Buchanan GR, Zimmerman S, Michaels LA, Kohan R, Berchtold W, et al. A prospective comparative study of 2540 infants and children with newly diagnosed idiopathic thrombocytopenic purpura (ITP) from the Intercontinental Childhood ITP Study Group. J Pediatr. 2003;143(5):605-8.

20. Buchanan GR, Adix L. Outcome measures and treatment endpoints other than platelet count in childhood idiopathic thrombocytopenic purpura. Semin Thromb Hemost. 2001; 27(3):277-85.

21. Buchanan GR, Adix L. Grading of hemorrhage in children with idiopathic thrombocytopenic purpura. J Pediatr. 2002;141(5): 683-8.

22. Page LK, Psaila B, Provan D, Michael Hamilton J, Jenkins JM, Elish AS, et al. The immune thrombocytopenic purpura (ITP) bleeding score: assessment of bleeding in patients with ITP. $\mathrm{Br} \mathrm{J}$ Haematol. 2007;138(2):245-8.
23. Chandra J, Ravi R, Singh V, Narayan S, Sharma S, Dutta AK. Bleeding manifestations in severely thrombocytopenic children with immune thrombocytopenic purpura. Hematology. 2006;11 (2):131-3.

24. Walker RW, Walker W. Idiopathic thrombocytopenia, initial illness and long term follow up. Arch Dis Child. 1984;59(4):316-22.

25. Robb LG, Tiedeman K. Idiopathic thrombocytopenic purpura: predictors of chronic disease. Arch Dis Child. 1990;65(5):502-6.

26. British Committee for Standards in Haematology General Haematology Task Force. Guidelines for the investigation and management of idiopathic thrombocytopenic purpura in adults, children and in pregnancy. Br J Haematol. 2003;120(4):574-96.

27. Holt D, Brown J, Terrill K, Goldsby R, Meyers RL, Heximer J, et al. Response to intravenous immunoglobulin predicts splenectomy response in children with immune thrombocytopenic purpura. Pediatrics. 2003;111(1):87-90.

28. Hemmila MR, Foley DS, Castle VP, Hirschl RB. The response to splenectomy in pediatric patients with idiopathic thrombocytopenic purpura who fail high-dose intravenous immune globulin. J Pediatr Surg. 2000;35(6):967-71.

29. Minkov M. Critical issues concerning splenectomy for chronic idiopathic thrombocytopenic purpura in childhood. Pediatr Blood Cancer. 2006;47(5 Suppl):734-6.

30. Mantadakis E, Buchanan GR. Elective splenectomy in children with idiopathic thrombocytopenic purpura. J Pediatr Hematol Oncol. 2000;22(2):148-53.

31. Dickerhoff R, von Ruecker A. The clinical course of immune thrombocytopenic purpura in children who did not receive intravenous immunoglobulins or sustained prednisone treatment. J Pediatr. 2000;137(5):629-32

Avaliação: Editor e dois revisores externos

Conflito de interesse: não declarado

Recebido: 18/06/2008

Aceito: $03 / 07 / 2008$ 